\title{
LOS PROCESOS DE DESARROLLO LOCAL EN LA REGIÓN NEA: UNA APROXIMACIÓN TEÓRICA Y EMPÍRICA
}

\section{Ana María Pérez}

Psicóloga (UNR). Magister en Ciencias Sociales (CLACSO). Investigadora de CONICET. Profesora titular Sociología de la Educación. Facultad de Humanidades (UNNE). Directora del Centro de Estudios Sociales- Rectorado (UNNE).

\section{PABlo Barbetti}

Licenciado en Relaciones Industriales (UNNE). Maestrando en Desarrollo Social. Facultad de Humanidades (UNNE). Profesor adjunto a cargo de las cátedras Administración y Selección de Recursos Humanos (Facultad de Humanidades-UNNE) y Seminario de Aplicación Metodológica (Carrera de Relaciones Laborales- UNNE). Investigador de la SECyT - UNNE con lugar de trabajo en el Centro de Estudios Sociales - Rectorado (UNNE). 
Procesos de desarrollo local en la Región NeA: Una aproximación teórica y empírica

\title{
Resumen
}

El artículo presenta resultados empíricos derivados de un estudio que analiza los proyectos de Desarrollo Local implementados en Argentina. En este caso particular, y luego de pasar revista a las perspectivas teóricas que sirven de sustento, se consideran las experiencias desarrolladas específicamente, en algunas localidades de la provincia del Chaco y Corrientes. La información empírica, con la cual se ha construido un mapa comparativo, da cuenta de una diversidad de estrategias que exponen, por un lado la amplia variabilidad de formas que se subsumen bajo esa categoría conceptual; junto con una cierta uniformidad en relación con el enfoque político-ideológico que se asume.

\begin{abstract}
The article presents empirical results derived from a study that analyzes the projects of Local Development implemented in Argentina. In this particular case, and after reviewing to the theoretical perspectives that use as sustenance, the experiences developed specifically are considered, in some localities of the province of the Chaco and Corrientes. The empirical information, with which a comparative map has been constructed, realizes of a diversity of strategies that expose, on the one hand the wide variability of forms that are subadded under this conceptual category; together with a certain uniformity in relation to the political-ideological approach that is assumed.
\end{abstract}




\section{Ana María Pérez y Pablo Barbetti}

PROCESOS DE DESARROLLO LOCAL EN LA REGIÓN NEA: UNA APROXIMACIÓN TEÓRICA Y EMPÍRICA

\section{INTRODUCCIÓN}

La posibilidad de pensar la integración entre políticas públicas y procesos de desarrollo local, se inscribe en el marco del concepto de globalización que ocupa un lugar central en los debates propios de las ciencias sociales, así como en el de la política en general. El término globalización, que comenzó a emplearse en la segunda mitad de los años 80, instaura, junto con la extensión del discurso neo-liberal, la idea de un mundo único, hechos que resultan concomitantes al derrumbe de "los socialismos reales".

A la vez, se profundizan las desigualdades entre los diferentes países y regiones, que sufren las consecuencias de las decisiones tomadas fuera de su ámbito geográfico, considerando que las posibilidades de alcanzar los deseados bienes del desarrollo dependen de la integración competitiva a los mercados mundiales. De este modo, y si bien la globalización induce a pensar en una deslocalización de los flujos, los conocimientos, las corporaciones, la información y la tecnología, lo local adquiere nuevas dimensiones, en tanto constituye el nivel donde se advierten, de modo más claro, los problemas que derivan de la apertura, la transformación del Estado y los procesos de integración; pero, también, donde se hace más evidente la necesidad de asociarse para competir en un mundo global, aunque preservando los valores y la calidad de vida de cada región y atendiendo, colectivamente, al compromiso ambiental.

A diferencia de la década de 1960, en la que el éxito de la planificación y las políticas de desarrollo local y regional se pensaban ligadas a las macro decisiones de carácter global o sectorial y al efecto de arrastre de los polos de desarrollo (Perroux, 1965) y los complejos industriales, durante las dos últimas décadas estas ideas han sido reemplazadas por la revalorización del territorio y lo local — sus aspectos históricos, culturales e identitariosel compromiso de los actores - los poderes públicos, los gremios, las cámaras, las empresas, las ONG, la escuela, la universidad, las asociaciones profesionales, los artistas-, sus capacidades para construir dinámicas de desarrollo y establecer relaciones y compromisos sinérgicos entre personas e instituciones. "La naturaleza del compromiso y la particularidad de la convergencia explican la originalidad del desarrollo local. No parecerse, sino cómo diferenciarse sustituye la puja por la equidad en el equipamiento" (Domecq, 2004). 


\section{CUADERNO}

Procesos de desarRollo local EN LA REgión NEA: UnA APROXIMACIÓN TEÓRICA Y EMPÍRICA

Esto se enmarca en una nueva concepción que no se centra sólo en la idea de la economía como derivándose del mismo crecimiento, sino en una noción de desarrollo como proceso que engloba, además, aspectos sociales, culturales y educativos. Se trataría de una estrategia, que se apoya en las capacidades locales para iniciar un proceso de construcción "desde abajo", entendiendo el desarrollo como un cambio integral en las condiciones de vida, motorizado por los mismos actores que serían beneficiarios (Rofman, 2004).

Por los años noventa, el PNUD presenta su primer informe sobre Desarrollo Humano, al que define como un proceso tendiente a la ampliación de oportunidades —en términos de libertad política, económica y social, creatividad y producción, auto-respeto y vigencia de los derechos humanos-, básicamente en lo que respecta a la calidad de vida, la adquisición de conocimientos y la salud y centrado en la formación de las capacidades y en el uso que se hace de ellos. En general, en todas las definiciones se incluyen principios generales tales como ampliación de oportunidades, fortalecimiento de la autonomía del sujeto y de las comunidades, integración social, equidad y participación.

\section{EL NUEVO PARADIGMA DE LAS POLÍTICAS Y LOS PROYECTOS}

Estas perspectivas configuran una suerte de nuevo paradigma en relación con las políticas sociales y los proyectos de desarrollo (Martínez Nogueira, 2004), anclados en los contextos locales y financiados por los organismos internacionales. En ellos asume centralidad una visión remedial que se traduce en programas compensatorios o de paliativo de los efectos negativos generada por la reestructuración económica y que derivan de las condiciones, acuerdos y negociaciones que proponen los organismos internacionales de crédito.

Por lo demás, estas propuestas pretenden establecer una perspectiva crítica en relación con la modalidad de pensar y aplicar las políticas sociales a la fecha, centralizadas y orientadas hacia supuestos universos homogéneos de destinatarios. En consecuencia, se articulan nociones de atención focalizada de necesidades, identificación específica de situaciones y beneficiarios, reconocimiento de heterogeneidades, descentralización, empowerment y participación activa de la sociedad civil. Asimismo, adquiere relevancia la noción de proyecto, fundamentada en los encuadres macro-económicos, en los mecanismos organizacionales y en los modos de gestión, aunque descuidando, nuevamente, la 


\section{Ana María Pérez y Pablo Barbetti}

PROCESOS DE DESARROLLO LOCAL EN LA REGIÓN NEA: UNA APROXIMACIÓN TEÓRICA Y EMPÍRICA

visión particularizada de los problemas en función de las distintas realidades nacionales y locales, los análisis históricos que dan cuenta de factores institucionales, actores o racionalidades operantes. En este marco se recurre a la noción de capital social, la que ha adquirido una cierta difusión en Latinoamérica, en particular a partir de la década de los años '90 (Putnam, 1994) y en la que se destacan los aspectos subjetivos, disposicionales o culturales, en términos de valores y actitudes propias de los individuos. Este capital social, derivado de los comportamientos de los actores, va a ser definido como condición y posibilidad del desarrollo político y económico de las sociedades periféricas (Klisberg, 2002), orientando de este modo las recomendaciones en torno al diseño de políticas sociales.

En el caso de América Latina, como consecuencia de la declinación del Estado de Bienestar y la puesta en práctica de las políticas de ajuste, se enfatizó el desarrollo de organizaciones pertenecientes al denominado "tercer sector" (Salamon, Anheier, 1995) —considerado como un espacio ubicado entre lo público y lo privado, o entre el Estado y el mercado, que agrupa a la totalidad de las organizaciones sin fines de lucro- ${ }^{1}$. Estas destacan la importancia de la acción colectiva pero desde un sentido despolitizado, asumiendo como principal tarea la transferencia de capacidades a los sectores populares con el objetivo de propiciar que, a través de su propio esfuerzo, logren mejorar sus condiciones de vida.

Lo que ha de destacarse en estos enfoques, es fundamentalmente su perspectiva a-histórica, basada en la consideración de la situación presente, de los objetivos que se espera alcanzar y el coste-beneficio que resulta de su aplicación, sin atender las particularidades de cada región o país o indagar acerca de su origen. El Banco Mundial (2001) con frecuencia suele realizar recomendaciones en torno de la aplicación de medidas que han logrado éxito en otras partes del mundo, con condiciones socio-económicas y culturales totalmente diferentes de la población beneficiaria, de las condiciones de la economía, o de las capacidades institucionales del Estado (Golbert, 2004:26).

Iniciativas de este tipo han sido llevadas adelante en numerosos países latinoamericanos (cfr. Llorens Alburquerque y Del Castillo, 2002; Clemente, 2003; Gallicchio, 2004), como Argentina; Brasil, México, Chile, Honduras, Perú, Uruguay. Si bien casi todas ellas pueden ser encuadradas en la categoría de desarrollo económico o local, se caracterizan por su gran diversidad en cuanto a su génesis, el ámbito de actuación, los objetivos, el alcan-
1 En el plano internacional la visión dominante del tercer sector corresponde al enfoque americano que la define como la esfera que agrupa la totalidad de organizaciones sin fines de lucro sin distinciones de ningún tipo. 


\section{CUADERNO}

Procesos de desarRollo local EN LA REgión NEA: UnA APROXIMACIÓN TEÓRICA Y EMPÍRICA

ce de las actividades, el papel desempeñado por los agentes sociales y los resultados obtenidos. Tal diversidad deriva de las diferencias que guardan entre sí las unidades subnacionales involucradas, con niveles de complejidad y desarrollo económico diferentes y que pertenecen asimismo a países con desiguales niveles de renta y grado de descentralización formal. Sin embargo, comparten una cierta debilidad o incompletad que se manifiesta en la mayoría de los procesos - ya se trate de programas promocionados por los organismos financieros internacionales o de experiencias generadas a nivel local一. Esta característica parece incidir en el modo en que se posicionan los actores frente a ellos, y que se traduce fundamentalmente en una pérdida de la motivación y frustración.

\section{LA ECONOMÍA SOLIDARIA: EL ENFOQUE EUROPEO}

Otra es la perspectiva del pensamiento europeo en relación con estas cuestiones, vinculadas con la integración y democratización de la sociedad, frente a la insuficiencia del modelo capitalista global. Se plantea, básicamente, en términos de un debate entre la economía social tradicional y la economía solidaria, caracterizada por una orientación menos micro-social y más macro-política que la primera; prestando atención a la articulación entre las dimensiones socio-política y socio-económica, a través de un enfoque dinámico e histórico. Esta especificidad implica una integración en el campo de las organizaciones denominadas de la economía social, que no se limita a aquellas sin fines de lucro, sino que comprende, además, a las cooperativas y las mutualidades. (Laville, 2004)

A la vez, y en lugar de definir proyectos, como modelos de transformación listos para ser puestos en práctica, se propone, más bien, la construcción colectiva de experiencias innovadoras, que pretenden dar respuesta a la nueva cuestión social (Rosanvallon, 1998; Castel, 1997) derivada de la crisis del Estado de Bienestar y la sociedad salarial. Afirmándose en la crítica del neo-institucionalismo y la despolitización de las propuestas centradas en la mera ausencia de fines de lucro y la desvinculación de la acción pública se otorga importancia central al principio de solidaridad social. La noción de economía solidaria tiene como objetivo la satisfacción del interés general o contribución al bien común y de respuesta a las demandas sociales expresadas por ciertas categorías de población (Laville y Sainsaulieu, 1997). Inspirada en los clásicos escritos de Mauss (1923) y 


\section{Ana María Pérez y Pablo Barbetti}

Procesos de desarRollo local EN LA REgIÓN NEA: UNA APROXIMACIÓN TEÓRICA Y EMPíRICA

Polanyi (1983), Perroux (1960) y Razetto Migliaro (1988), se plantea la posibilidad de pensar nuevas formas de organización no capitalista del desarrollo, tomando a la democratización como condición concomitante.

Estos autores proponen una definición extensa de economía distinguiendo tres principios (Laville, 2004): el mercado, que permite un encuentro entre oferta y demanda de bienes y servicios con la finalidad de intercambio a través de la fijación de precios, la redistribución, asociada a la existencia de una autoridad y procedimientos mediante los que se establecen reglas de extracción y asignación de la producción y la reciprocidad, entendida como la relación establecida entre grupos y personas basada en la voluntad de manifestar el lazo social entre las partes; en tal sentido se opone al intercambio mercantil y se distingue del intercambio redistributivo, ya que no existe un poder central. La hibridación de estos principios resultaría en modos de protegerse contra la marginación; mientras que en la dimensión socio-política, en tanto, se enfatizan el conjunto de interacciones entre los poderes públicos y las iniciativas de la sociedad civil que se traducen en efectos mutuos.

A diferencia del planteo anterior, las propuestas que se llevan adelante no encuentran su origen en las deficiencias que presentan algunos públicos focalizados e identificados con respecto a una supuesta normalidad, sino en la interrogación colectiva sobre lo que cotidianamente viven los interesados. Por otra parte, su interés principal es que los problemas planteados no se resuelven en la vida privada, si no en la esfera pública. De este modo, en lugar de constituirse en paliativos contra el desempleo, proponen una reflexión acerca de la naturaleza del lazo social y las finalidades del intercambio económico; se trata, en definitiva, de una recomposición de las relaciones entre lo económico y lo social.

Pero la situación europea y latinoamericana no es fácil de comparar, y existen importantes diferencias entre las posibilidades de instaurar servicios de proximidad (Eme y Laville, $1994)^{2}$ con calidad social y política en el viejo continente y en nuestra región, en la que es preciso, primero, resolver necesidades de nutrición y servicios elementales básicos y donde ningún cambio resulta posible sin que se produzcan, antes, profundas modificaciones en lo económico, lo político y la justicia.
2- Los servicios de proximidad constituyen una forma alternativa de atención a determinados problemas sociales en los que los servicios prestados son concebidos como una forma de reciprocidad que permite una construcción conjunta de la oferta y la demanda a través de espacios públicos en los que prima la cercanía

(proximidad) y en los que, una vez creados, los servicios se consolidan por la hibridación entre diferentes tipos de recursos - mercantiles, no mercantiles y no monetarios-. 


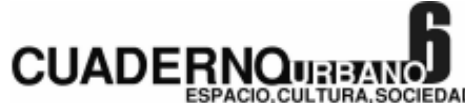

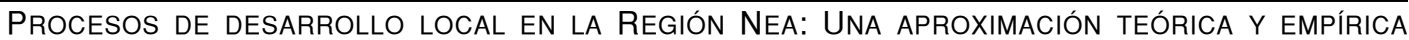

\begin{abstract}
3- En lo sucesivo trabajamos con la sigla DL al referirnos al Desarrollo Local. El corpus de ideas que constituyen la propuesta del desarrollo local congrega, no siempre de manera sistematizada, un espectro de líneas de pensamiento ideológicamente variada (Choconi, S. 2003) y, tal sentido es posible encontrar aproximaciones muy diferentes asociadas a determinados enfoques: como desarrollo económico, como estímulo para la participación, como municipalismo, como mecanismo de ordenamiento territorial, entre otros.
\end{abstract}

4- Este trabajo forma parte de un proyecto de investigación más amplio denominado "TRABAJO, DESARROLLO, DIVERSIDAD -

Una investigación sobre

metodologías y políticas de desarrollo local con acento en la generación de empleo / trabajo

ingresos" ejecutado por una $R E D$ de Investigadores pertenecientes a instituciones de diferentes regiones del país con el financiamiento de la Secretaría de Ciencia y Tecnología de la Nación.

\section{LOS PROYECTOS DE DESARROLLO EN LA REGIÓN NEA: EL CASO DE CHACO Y CORRIENTES}

Conformada por las provincias de Formosa, Chaco, Misiones y Corrientes constituye una región deprimida y periférica al eje central de la producción pampeana. La zona rural continua siendo importante, con urbanizaciones a la vera de los ríos, resultado, en parte, de las migraciones internas hacia esas ciudades, por lo que se constituye en zona de expulsión con mucho dinamismo demográfico (Panaia, 2004). Por su parte, las provincias de Chaco y Corrientes, marco de nuestro estudio, se encuentran ubicadas muy próximas entre sí, separadas por el río Paraná y unidas por el Puente General Belgrano, lo que facilita, al menos entre las ciudades de Resistencia y Corrientes, el flujo permanente de personas. Ambas, presentan altos índices de pobreza, NBI y desnutrición infantil y en general, indicadores sociales preocupantes. Asimismo, las tasas de participación laboral son comparativamente menores que en otras provincias siendo las principales fuentes de ocupación la administración pública, el comercio y el servicio doméstico. Si bien tienen muchos aspectos en común guardan, sin embargo, importantes diferencias en los culturales, que derivan, fundamentalmente, de la ausencia de migración de ultramar a fines del siglo XIX en la provincia de Corrientes, mientras que en Chaco la conformación del territorio, se hizo — casi tres siglos después - a partir de los colonos que vinieron a instalarse en él.

En los últimos años, y bajo la denominación general de Desarrollo Local ${ }^{3}$, en ambas provincias, se ha puesto en marcha un conjunto de experiencias, de las que participan municipios de diferentes categorías y perfil productivo, pertenecientes igualmente a zonas rurales y urbanas y que, en conjunto, constituyen un universo heterogéneo. Abarcando un variado espectro de situaciones de escalas diferentes que configuran un continuo que incluyen las siguientes situaciones: a) proyectos más o menos acotados, generados desde algún sector específico (municipio, organización de la sociedad civil con diversos grados de articulación con otros actores sociales), b) procesos más amplios situados a nivel municipal (bajo la conducción del gobierno local y en el que se involucra una mayor cantidad de sectores de la comunidad) y, c) Procesos a nivel microrregional (a partir de la asociación de varios municipios, en los que interviene el Gobierno Provincial con un rol significativo).

En este contexto, en el presente artículo ${ }^{4}$ nos proponemos plantear algunas reflexiones derivadas del análisis de los proyectos de desarrollo implementados en la región NEA: la 


\section{Ana María Pérez y Pablo Barbetti}

PROCESOS DE DESARROLLO LOCAL EN LA REGIÓN NEA: UNA APROXIMACIÓN TEÓRICA Y EMPÍRICA

perspectiva teórico-metodológica en la que se encuadran, los actores que participan, los objetivos que se explicitan y los principales resultados que se han alcanzado. La información -derivada tanto de datos primarios como secundarios ${ }^{5}$ - ha sido procesada mediante el análisis factorial de correspondencia ${ }^{6}$, técnica que permite revelar el cuadro de propiedades del conjunto de casos, los que pueden ser caracterizados a partir de tales propiedades, configurando distintos colectivos o clases; $y$ fundamentalmente identificar grupos conformados a partir de la asociación de un conjunto de variables significativas. Ver Gráfico 1.

El universo de análisis estuvo integrado por las siguientes localidades, de la provincia del Chaco, Puerto Tirol, Presidencia de la Plaza, Machagai, Quitilipi, Villa Angela, Villa Río Bemejito; de la provincia de Corrientes: Colonia Liebig, Carlos Pellegrini, Santa Lucía, Goya, Curuzú Cuatiá, Monte Caseros y Corrientes (Capital). Ver Mapa l. La información relevada en cada una de ellas fue traducida a un sistema de categorías ${ }^{7}$ y sus correspondientes subdimensiones; luego se construyó una tabla de filas por columnas, en la que cada fila representaba una localidad y las columnas, las distintas categorías descriptivas. El procesamiento de esta tabla con la técnica señalada permitió caracterizar los procesos de desarrollo emprendidos y organizar un mapa comparativo del conjunto de casos.

Según éste y en una primera aproximación, es posible diferenciar estas experiencias en relación con su origen, ya sea que deriven de iniciativas privadas o gubernamentales y que se encuentren ubicadas en el nivel local/municipal/

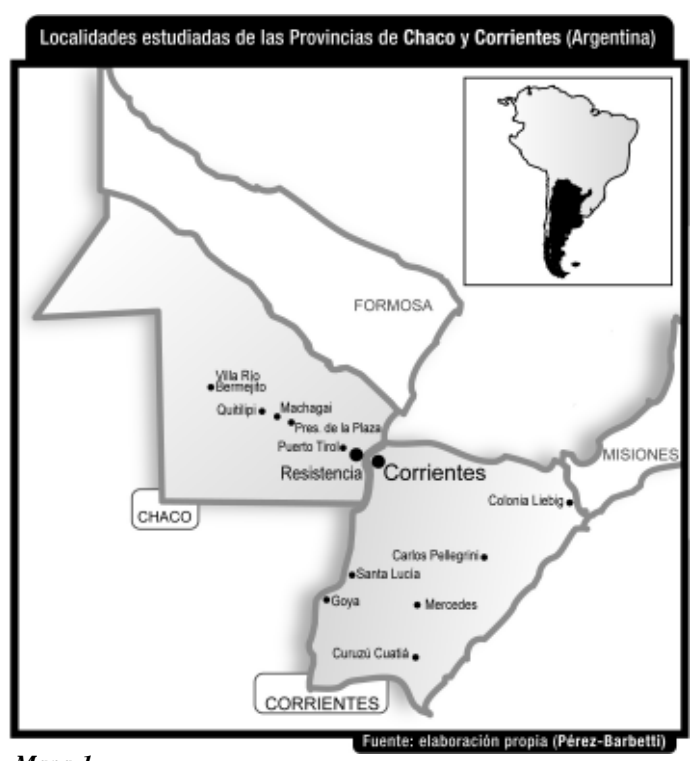

Mapa 1
5- Los datos primarios derivaron de entrevistas realizadas tanto a funcionarios municipales como pobladores de cada una de las localidades. Los datos secundarios corresponden a información documental de cada uno de los procesos (Planes de Desarrollo; Registros de Reuniones, etc.) así como otros documentos oficiales del municipio y datos estadísticos.

6- El análisis factorial de correspondencias es una metodología descriptiva que transforma un conjunto de medidas individuales en factores que describen la máxima variabilidad, dando cuenta del modo cómo se organizan modo como se organizan
colectivamente los datos en el espacio multidimensional mediante el análisis simultáneo de un gran número de variables cualitativas $u$ ordinales- $y$ sus relaciones, privilegiando algunas y proporcionando una estructura y proporcionando una estructura
gráfica de los datos. Tratando adecuadamente las co-ocurrencias observadas permite la elaboración de tipologías que son reproducibles independientemente del observador, facilita la comparación de todas las unidades de observación, a través de todas las modalidades de las características observadas.

7- Algunas de las principales categorías que fueron consideradas fueron las siguientes: momento histórico en que surge la experiencia, objetivos, origen y duración del proceso, origen del

financiamiento de la experiencia, estado de avance y principales estado de avance y principales
resultados considerados por los resultados considerados por los
participantes, actores y/ sectores participantes, metodología utilizada en las diferentes instancias del mismo. 
CUADERNQuiand

Procesos de desarRollo local en la Región NeA: UnA APROXIMACIÓN TEÓRICA Y EMPíRICA

\section{8- Nos referimos especialmente descentralización de competencias del gobierno nacional a los municipios y desconcentración en la prestación de servicios básicos así como a las privatizaciones y transferencias de funciones tradicionales estatales al ámbito de la sociedad civil y empresas.}

Grafico 1

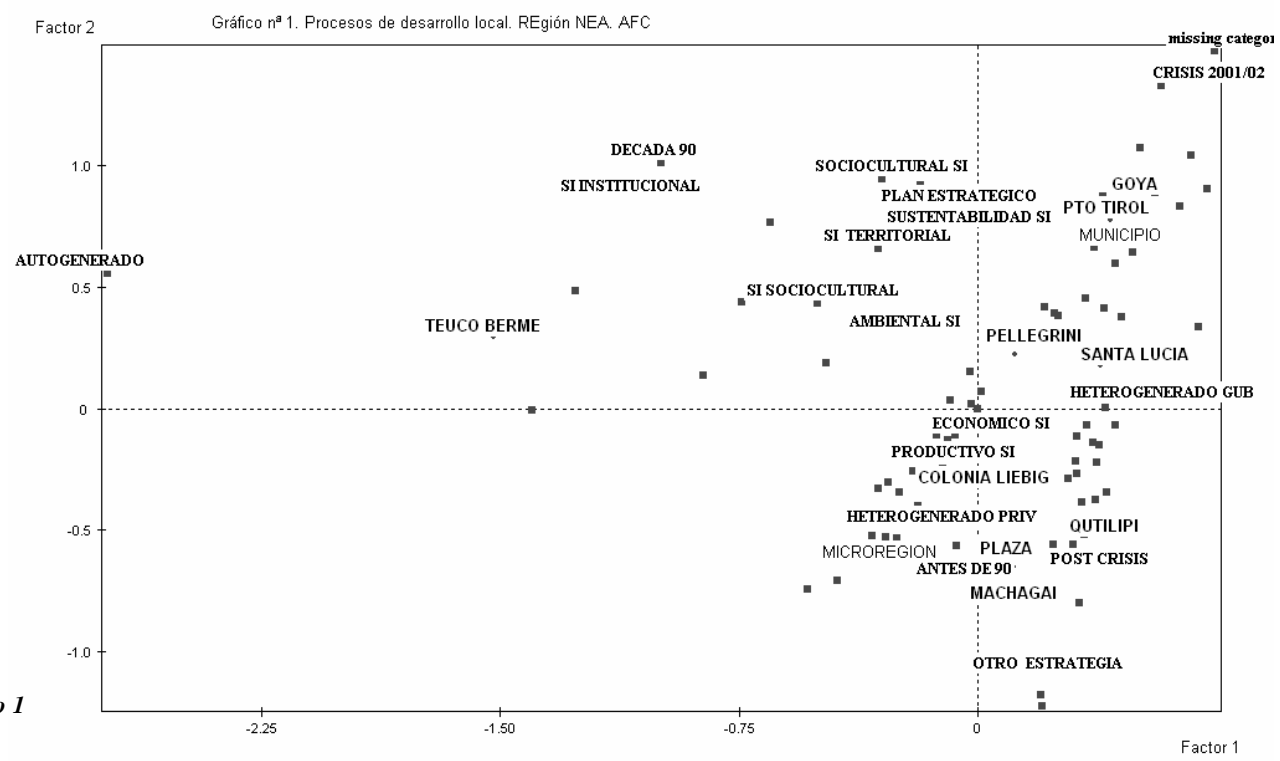

provincial o bien respondan a decisiones asumidas en el ámbito nacional o internacional. Otro elemento que permite establecer distinciones deriva del momento de su implementación, y se constituye en un aspecto relevante para comprender la orientación asumida: durante los años '90, en el punto más alto del auge neo-liberal en nuestro país, coincidente con un contexto de debate y re-definición de la relación Estado-Sociedad ${ }^{8}$, en los inicios de la década del 2000 con la gran crisis económica, financiera e institucional; o bien, en etapas previas o posteriores, en la llamada pos crisis.

\section{La década de los 90 y la crisis: el planeamiento estratégico}

Coincidente con los enfoques que privilegiaban la descentralización del Estado y la perspectiva del desarrollo social, durante la década de los `90, y el momento más álgido de la 


\section{Ana María Pérez y Pablo Barbetti}

PROCESOS DE DESARROLLO LOCAL EN LA REgIÓN NEA: UNA APROXIMACIÓN TEÓRICA Y EMPÍRICA

crisis, se encuadran los Planes Estratégicos buscando atender a las condiciones de vulnerabilidad social de algunos sectores; pero, fundamentalmente, contribuir a consolidar situaciones de gobernabilidad en el interior de las regiones. Considerada una metodología innovadora, constituye uno de los instrumentos más difundidos y aplicados en las intervenciones orientadas al desarrollo. En ellos se propone un trabajo que, incluyendo la participación de diferentes actores - tanto públicos como privados-, se oriente hacia un diagnóstico del presente e identifique un escenario futuro deseable, para luego definir las estrategias para alcanzarlo. En las provincias bajo análisis, las localidades que realizaron experiencias de este tipo durante este período fueron: Villa Río Bermejito, Puerto Tirol, Carlos Pellegrini, Goya, Corrientes y Santa Lucía. A partir de las consideraciones de los informantes claves de estos procesos, se advierte la diversidad de objetivos que aparecen destacados y/o valorizados en cada caso. En general, con estos procesos se pretendía, básicamente, la promoción del desarrollo económico y productivo, a partir de la definición o redefinición de los perfiles locales, partiendo de la identificación de sus ventajas comparativas, la generación de nuevas iniciativas económicas que potenciaran la creación de trabajo y empleo (tanto en el sector económico primario como en la actividad industrial, comercial y de servicios). Si bien estos proyectos estaban anclados, siempre, en el interés por alcanzar algunos de los beneficios del crecimiento económico, las alternativas a las cuales abonan son variadas incluyéndose objetivos vinculados con lo territorial planeamiento, ordenamiento y gestión del territorio-, lo sociocultural, la sustentabilidad, y lo institucional; acordando de este modo con la lógica propuesta por el modelo orientado a privilegiar las particularidades locales en cada caso. En este contexto resultan interesantes los lineamientos que plantea el Plan Estratégico de Puerto Tirol (Chaco), que independientemente de su puesta en práctica, en el documento delinea la intención de avanzar en acciones de turismo religioso; asimismo se diseñaron programas orientados a desarrollar el sector agrícola minifundista, promover el nacimiento y la consolidación de micro-emprendimientos locales y generar el marco para atraer y favorecer la radicación de nuevas industrias ${ }^{9}$. Una experiencia similar, puesta en marcha entre 1998 y 2000, se realizó en la localidad de Goya (Corrientes) ${ }^{10}$, en parte a partir de la identificación de la pérdida de valor y rentabilidad de algunos de sus productos económicos tradicionales (tabaco, algodón y maíz), y que derivó en el intento de establecer acuerdos en la localidad, en particular entre el estado municipal y el sector productivo, con vistas a la diversi-
9- En esta línea, en los últimos años, se realizaron gestiones para la instalación de empresas de capitales nacionales - una fábrica de alfajores, otra de elaboración de fibras de vidrio y una aceitera- $e$ internacionales, específicamente, una empresa textil de capitales brasileños.

10- Actualmente, impulsados desde el nuevo equipo de gobierno municipal se está reeditando un proceso de planificación estratégica participativa, con algunos ajustes en la metodología y modificaciones en los actores convocados. El objetivo, en este caso, es establecer consensos sobre una visión de la comuna hacia el 2016. 


\begin{abstract}
11- Se trata de una experiencia generada desde un grupo de empresarios foráneos erradicados en el lugar e impulsada por el gobierno provincial. La falta de caminos y el deterioro de la economía local con la consiguiente emigración de sus habitantes, estratégicamente fueron convertidas en fortalezas. Así, la distancia a los grandes centros urbanos y las dificultades de acceso favorecieron su conservación como reserva natural; por ello los pobladores que permanecieron en la zona que permanecieron en la zona localidad preservando su flora y fauna, hasta llegar a ser Sitio Ramsar y lugar de interés para el ecoturismo.
\end{abstract}

12- El mismo incluyó varios programas específicos: de adaptación a la nueva situación (acompañamiento psico-social a los afectados por la

relocalización); b) Nuevos

Asentamientos (para crear recrear y mejorar los

asentamientos existentes); c)

Desarrollo Regional y
Diversidad Cultural (que buscó

favorecer el desarrollo

sustentable a través de

fortalecimiento de la base económica y sociocultural); d) Sustentabilidad Ambiental (se proponía atenuar los efectos del impacto que produzca el movimiento).

13- Profesionales de la Universidad Nacional del Nordeste (UNNE), la UTN Facultad Regional Resistencia-, la Universidad Nacional de

Formosa (UNAF) y la FLACSO aparecen como técnicos en diferentes instancias del proceso. ficación de las líneas de producción. Mientras que en Colonia Carlos Pellegrini (Corrientes) se encaminaron las estrategias tendientes a la configuración de un enclave de turismo ecológico destinado a población de altos recursos ${ }^{11}$. Por su parte, en la experiencia de la ciudad de Corrientes, gran parte de los proyectos generados, en el marco de la planificación estratégica, se orientaron al establecimiento de pautas programáticas para el mejoramiento de la esfera institucional y social, y vinculados con las dimensiones de la comuna, su carácter urbano y el contexto político en el que se desarrolló la experiencia.

La localidad de Villa Río Bermejito en la Provincia del Chaco constituye un caso particular: este proceso se origina a partir de la devolución — por parte del gobierno provincialde 150.000 hectáreas de reserva a la Comunidad Toba del Interfluvio Teuco-Bermejito, luego de una larga etapa de reclamos reivindicatorios de esta comunidad indígena. Esta situación, que supuso la necesaria re-localización de la población criolla allí asentada, impulsó la concreción de un proyecto integrado de planificación estratégica, en el que se incluyó, además de la consideración de los aspectos económicos y el tratamiento de la sustentabilidad ambiental (también considerados en las otras experiencias mencionadas), la atención a la diversidad cultural de la población participante ${ }^{12}$.

Otro rasgo que permite caracterizar estas experiencias es que han surgido, especialmente a partir de iniciativas generadas desde los organismos oficiales (nacional, provincial y municipal) en coordinación y asociación con otras instituciones de apoyo técnico (Universidades Nacionales ${ }^{13}$, INTA, INTI) y/o financiero (tanto nacionales como internacionales ${ }^{14}$ ); aún cuando se identifican otros casos en los que su realización derivó de la determinación de diferentes actores de la sociedad civil local, bien se trate de ONG ${ }^{15}$, o de empresarios y sus organizaciones ${ }^{16}$.

Sumado al interés por lo local, el papel que se otorga a la participación deviene un elemento relevante cuando se trata de diferenciar este modo de promover el desarrollo, del paradigma clásico. En los casos comprendidos en este estudio, en general, se incluye un conjunto de estrategias mediante las cuales se prevé la instauración de espacios participativos, concretados, en su mayoría, durante la etapa de formulación, aunque con diferentes grados de formalidad - mesas de concertación, consejos consultivos, talleres, asambleas - y/o recurriendo a técnicas específicas tanto en el trabajo de diagnóstico como de planificación. 


\section{Ana María Pérez y Pablo Barbetti}

PROCESOS DE DESARROLLO LOCAL EN LA REGIÓN NEA: UNA APROXIMACIÓN TEÓRICA Y EMPÍRICA

Aunque, desde la mirada de los distintos actores locales estos espacios son valorados positivamente, en especial porque devienen en una instancia de encuentro - y reflexiónque favorece la construcción de visiones compartidas sobre el futuro deseado para la localidad, también son evidentes algunos puntos de tensión que se generan en torno de ellos. Así, mientras que los referentes técnicos y políticos identifican entre los principales aspectos deficitarios la falta de interés de ciertos sectores ${ }^{17}$, o la imposibilidad de que se mantengan durante todo el proceso, los miembros de las organizaciones sociales, como contrapartida, señalan el carácter restringido de las convocatorias o la inadecuación de las estrategias de difusión y sensibilización para atraer, de manera efectiva, a ciertos colectivos sociales ${ }^{18}$. En consecuencia, y lejos de conformarse como una posibilidad de participación genuina, en la que hallen expresión los distintos grupos o estratos, se configuran, más bien, como lugares formales de encuentro entre los técnicos extra-locales expertos/consultores en la gestión de proyectos, que no conocen la problemática local ni son actores reconocidos por la comunidad, dado el carácter discontinuo y fragmentado de su intervención- y algunos sectores privilegiados de la localidad; un entrevistado, ejemplificando de modo muy claro esto, señalaba: "El menchaje sirve para hombrear bolsas"(Tirol).

El aprovechamiento dado a los productos de estos procesos ha sido variable y a pesar de que en algunos de ellos han quedado registros documentales de estos diagnósticos Goya, Puerto Tirol, Corrientes - no siempre impactaron en la agenda municipal ni dieron lugar a orientaciones en la definición de las políticas y la elaboración de los programas e incluso, en ocasiones, ni siquiera fueron implementados (Corrientes). Los actores participantes destacan una cierta tendencia a "quedarse en la instancia de diagnóstico", que obedecería, fundamentalmente, a cambios producidos en la gestión de gobierno y que impedirían su continuidad; a la necesidad de atender a problemas coyunturales; la insuficiencia de recursos humanos calificados, en las estructuras municipales, que permitan sostener las experiencias en el tiempo; la falta de recursos financieros o de apoyo político para su implementación; pero también, la debilidad de los acuerdos y alianzas interinstitucionales que se generan en tales experiencias.

En relación con los resultados, aquellos que son destacados por los diferentes actores consultados, difieren en función de las características particulares de cada municipio y
14- Entre los programas de Cooperación Internacional que financiaron algunas experiencias se encuentran, PNUD y Red Urbal - Programa de Cooperación Descentralizada de la Comisión Europea para Europa y América Latina.

15- El antes mencionado proyecto integrado TeucoBermejito fue impulsado desde la Asociación MEGUESOXOCHI, representante de la Comunidad Indígena Toba de la Provincia del Chaco.

16- Como fue el caso específico de la ya citada Colonia Carlos Pellegrini.

17- También esto constituye un aspecto que varía en cada caso, mientras que en algunas experiencias se alude a la falta de intervención del sector empresarial: "El sector privado negocia de modo bilateral con el municipio o incluso por encima del mismo, según necesidades" (Puerto Tirol), en otros casos se demanda una participación más activa de otros sectores como el Estado (Villa Río Bermejito) y las ONG (Goya).

18 Las comunidades aborígenes los trabajadores de áreas rurales así como las organizaciones vinculadas a organizaciones sindicales o movimientos piqueteros, devienen en grupos que, en las prácticas

participativas, para los referentes técnicos y políticos aparecen como sectores con un importante grado de complejidad (en el momento de pensar estrategias momento de pensar estrategias
tanto para su convocatoria como para gestionar potenciales conflictos posteriores derivados de sus intervenciones). 
19- En este grupo también se ha incluido un caso, Colonia Liebig, cuyo proceso se inició antes de los años 90 y que, en consecuencia, no se encuadra en las características del grupo anterior. de las circunstancias a partir de las cuales se iniciaron estos procesos. En el caso del Teuco Bermejito, se destacan los aspectos vinculados con procesos de movilización, cohesión social y cuestiones culturales porque, aún cuando la integración de la comunidad aborigen está lejos de resolverse, la experiencia posibilitó instalar el tema en la agenda de los gobiernos provincial y municipal, generar instancias de diálogo entre diferentes sectores e incluso avanzar concretamente en algunas de las demandas históricas de este grupo. En los restantes casos, el énfasis está puesto en los resultados de orden económico e institucional; en particular aquellos que se consideran asociados al desarrollo regional - Santa Lucía - la obtención de inversiones extranjeras - Puerto Tirol y Colonia Carlos Pellegrini- o el aprovechamiento de los recursos públicos nacionales y provinciales acompañando el proceso. Sin embargo, y a pesar de que, como consecuencia de tales planes, se realizaron algunas gestiones concretas de mejoramiento en las comunas —ordenamiento catastral, ampliación de la cobertura de servicios de infraestructura, asistencia y promoción de emprendimientos asociativos - esta tendencia no se vio reflejada en un aumento análogo en los indicadores de calidad de vida -empleo, salud, educación-. En resumen, estas experiencias de vinculación y asociatividad entre Estado, sociedad civil y sector privado, más que transformaciones significativas, parecen haber reproducido el tipo de relaciones pre-existentes y características de las regiones periféricas.

\section{La pos-crisis y los programas sociales}

Con estrategias y perspectivas diferentes a los casos anteriores, se agrupa un conjunto de experiencias en otras localidades que tienen en común haberse iniciado/desarrollado después de la década del 90 y la crisis consiguiente ${ }^{19}$.

Al tiempo que avanzaba, al menos a nivel de discurso, el cuestionamiento a las políticas neo-liberales, se profundizaban la exclusión y las desigualdades, los grupos dirigentes pasaban por una fuerte crisis de representatividad y los conflictos sociales recrudecían, se concibe un conjunto de Programas Sociales, que, si bien son diseñados por los organismos centrales se implementan a nivel local, especificando la orientación que va a asumir el modelo de desarrollo en este período. Entre ellos, el Plan Jefas y Jefes de Hogar constituye una referencia necesaria, en tanto se ajusta al esquema administrativo y de gestión 


\section{Ana María Pérez y Pablo Barbetti}

PROCESOS DE DESARROLLO LOCAL EN LA REGIÓN NEA: UNA APROXIMACIÓN TEÓRICA Y EMPÍRICA

propio de esta orientación y que es reproducido, luego, en la mayoría de las políticas y programas que surgen con posterioridad ${ }^{20}$. En general, estos programas se han asentado en objetivos de tipo compensatorio y de contención social tendiendo - al igual que los Planes Estratégicos del período anterior - a favorecer la gobernabilidad; quizá precisamente por ello y habida cuenta de la alta conflictividad social que caracterizaba el período, su puesta en marcha fue acompañada con estrategias tendientes a promover la participación de los distintos actores involucrados. Es en este contexto donde se ubica el sur gimiento de los Consejos Consultivos Locales ${ }^{21}$ (Clemente, 2005), estableciendo un quiebre en el modo de operar de los programas sociales hasta la fecha. Derivados, en parte, de la crisis y respondiendo a los ejes centrales del modelo innovador de gestión social ${ }^{22}$ propuesto por los organismos internacionales, los Consejos Consultivos Locales aparecen, en los municipios, con una doble función: por un lado, contribuir al proceso de descentralización estatal, efectuando algunas tareas específicas a nivel local (administrar las altas y bajas de los beneficiarios de los distintos programas o prestar los avales requeridos a los diferentes proyectos) y, por otro, constituir espacios de participación para los ciudadanos, en los que se revise el grado de ajuste entre las ofertas propuestas y las necesidades de los beneficiarios y, además, se audite la transparencia, eficacia y eficiencia del uso de los recursos públicos.

El eje central de esta propuesta, al igual que para los planes estratégicos, se asienta en las posibilidades de establecer articulaciones e interrelaciones entre los actores de la comunidad. En los municipios incluidos en este grupo -Quitilipi, Presidencia de la Plaza y Machagai en la Provincia del Chaco; Colonia Liebig, Monte Caseros y Curzú Cuatiá, en Corrientes - esos consejos han servido como estímulo para las organizaciones de la sociedad civil, favoreciendo la generación de proyectos socio-comunitarios y productivos. Efectivamente, nuestros entrevistados dan cuenta de experiencias asociativas de las cuales destacan un conjunto de aspectos positivos tanto en el plano comunitario, como en el territorial y financiero, así, en Curuzú Cuatiá, el CCL, conformado por instituciones de bien público y ONG, conjuntamente con el municipio, además de establecer acciones para lograr un mejor aprovechamiento del Plan Jefas y Jefes de Hogar, promovió - más allá de los subsidios - la concreción de proyectos socio-comunitarios en el área de salud y educación y de emprendimientos productivos. Asimismo, en Monte Caseros, y frente a la crisis del mo-
20- A modo de ejemplo se pueden mencionar algunos de los programas que operaron en ambas provincias en dicho período: Programa Social Agropecuario (PSA), Programa INCLUIR (Jóvenes), Programa de Atención a menores en Riesgo (PROAME), Plan de Desarrollo Local y Economía Social Manos a la Obra, Programa REDES, PROMEBA (Programa de Mejoramiento Barrial).

21- Los Consejos se han constituido en distintos niveles: nacional, provincial, local y barrial, con funciones $y$ características específicas en cada caso. Los Consejos Consultivos Municipales, de acuerdo con la normativa, son los que están en contacto directo con la realidad del territorio y las necesidades de la población.

\footnotetext{
22- El supuesto central del denominado "modelo innovador" es que lo que debe determinar la organización y gestión de un programa es su intento de maximizar el impacto y la eficiencia interna (a diferencia del tradicional que, asumiendo el impacto como un dato, tenían como función objetivo tratar de lograr la máxima eficiencia (mínimo costo) y cobertura. La participación de la sociedad civil como garante del ajuste entre las ofertas del programa $y$ las necesidades de los beneficiarios y con un rol de auditor del uso de los recursos aparecen como variables centrales de este modelo. CEPAL (1998)
} 
CUADERNOM

Procesos de desarRollo local en la Región NeA: UnA APROXIMACIÓN TEÓRICA Y EMPíRICA

23 Los referentes de las firmas locales así como sus asociaciones más

representativas (Cámaras

Empresariales) parecen no

encontrar interés en estos

espacios, en tanto negocia de modo bilateral con el municipio o incluso por encima del mismo, según necesidades.

24- Con frecuencia, las ONG se presentan con un bajo grado de formalización institucional, insuficiente disponibilidad de recursos humanos técnicos $y$ financieros, así como con dificultades para acceder a la información acerca de los

distintos programas sociales, sus objetivos, alcances $y$ beneficios.

25- No hay una representación directa de sus integrantes si no que la misma es institucional (bajo el criterio de autoridad y jerarquía).

26- Dependiente del Ministerio de Economía, Obras y Servicios Públicos. Gobierno del la Provincia del Chaco.

\section{7- La Unión de Municipios del Sudoeste Chaqueño fue la primera en constituirse como} Micro- Región.

28- Ley 5174. Sistema Provincial de Planificación y Evaluación de Resultados. Programa de Regionalización y Desarrollo delo agrícola vigente en la región, propició instancias de sensibilización y capacitación y la construcción de alianzas para la generación de experiencias de microemprendimientos en líneas no tradicionales, como el turismo rural o la producción citrícola y apícola.

Pero, tal como lo señalan los entrevistados, ha sido precisamente en esta búsqueda de vinculaciones y alianzas donde reside, al mismo tiempo, el punto más débil de su funcionamiento; debido, en parte, a la ausencia del sector empresarial ${ }^{23}$, o a las características que detentan algunas organizaciones sociales en estas localidades ${ }^{24}$, o sus limitaciones para acreditar una representación efectiva ${ }^{25}$, y, finalmente, la persistencia de relaciones clientelares establecidas entre la dirigencia política, los punteros de las organizaciones de base y los beneficiarios.

Contemporáneos a estas estrategias —organización de Consejos Consultivos e implementación de Programas Sociales - se conformaron organismos de cooperación intermunicipal, que devinieron en formas asociativas específicas destinadas a constituir espacios territoriales de articulación para la promoción del desarrollo local y regional: las microrregiones; que incluían entre sus propósitos la resolución de problemas en otra escala y el fortalecimiento del poder de negociación de los municipios intervinientes frente a los organismos de gobierno provincial y nacional, robusteciendo la lógica territorial horizontal de los actores estatales. Si bien en un comienzo surgieron como iniciativas aisladas, entre algunas comunas y con distinto grado de institucionalización, progresivamente se convirtieron en políticas provinciales.

En la provincia del Chaco, este proceso fue gestionado desde la Secretaría de Planificación y Evaluación de Resultados ${ }^{26}$, mediante un Programa de Regionalización y Desarrollo Local, cuyas primeras acciones se realizaron en 2000, con la constitución de la Umdesoch $^{27}$. Hacia finales del 2002, se instituyó el Sistema Provincial de Planificación y Evaluación de Resultados (SSPER) que sirvió de base para la planificación estratégica ascendente de políticas públicas, teniendo como ejes centrales: la descentralización y articulación (entre los actores sociales y territoriales en el diseño e implementación de las Políticas Públicas) y el mejoramiento de la gestión pública a partir de la moderniza- 


\section{Ana María Pérez y Pablo Barbetti}

PROCESOS DE DESARROLLO LOCAL EN LA REGIÓN NEA: UNA APROXIMACIÓN TEÓRICA Y EMPÍRICA

ción del estado ${ }^{28}$. Actualmente existen ocho microrregiones ${ }^{29}$ formadas por voluntaria asociación de los municipios (68), con procesos en variadas etapas de maduración: Umdesoch, Centro, Sudoeste II, Oriental, Metropolitana, Impenetrable, Norte y Centro Oeste.

Inscriptas en el modelo de planificación estratégica difundido en la década del 90, actualmente, en cada una de ellas, se trabaja en la definición de un perfil productivo propio, a través de un diagnóstico participativo, información que, por lo demás, las habilita a recibir algunos de los programas promovidos desde los Ministerios de Trabajo y Desarrollo Social. También se reconocen tareas de sensibilización institucional y comunitaria y actividades de capacitación destinadas a gerentes y promotores locales o a funcionarios públicos. Las dificultades que se identifican en torno de ellas - tanto en el proceso de conformación como de gestión- derivan, fundamentalmente, de los conflictos y divergencias que se vinculan con el origen partidario de los responsables de los distintos municipios o la coexistencia de visiones diferentes en relación con las políticas públicas de desarrollo ${ }^{30}$.

En el caso de la Provincia de Corrientes, en el marco de estas estrategias, se pueden identificar dos visiones, la de la Secretaría de la Pequeña y Mediana Empresa (SEPYME) y la de la Subsecretaria de Asuntos Municipales, con algunas diferencias en sus líneas de intervención. Desde esta última, se definieron 19 microrregiones ${ }^{31}$, de acuerdo con la proximidad física y las posibilidades de comunicación entre los municipios que lo integran. El objetivo central de su constitución ha estado asociado con la necesidad de identificar problemas comunes y elaborar propuestas para el diseño de las políticas públicas del gobierno provincial. En el marco de las localidades estudiadas, y si bien se ha avanzado en la identificación de diagnósticos regionales, tales estrategias son incipientes y no han generado espacios de institucionalización adecuados que permitan la retroalimentación de esta información a los distintos actores tendiente al diseño de políticas públicas. La SEPYME, por su parte, partiendo del marco normativo de las Agencias de Desarrollo $^{32}$ divide a la provincia en cinco nodos ${ }^{33}$, definidos a partir de criterios de proximidad física, caracterización agro-ecológica de los departamentos y formas de relacionamiento. Cada uno de estos nodos tiene una Agencia de Desarrollo con sede en un municipio; asociadas al enfoque economicista del DL, estas agencias, actualmente

\begin{abstract}
29- De acuerdo a los materiales de difusión, desde el programa el concepto de desarrollo es entendido dentro de un contexto más amplio que el de la economía, como subjetivo, valorativo, endógeno, sistémico y complejo, dependiente de la autoconfianza colectiva de crear y recrear recursos y de actuar solidariamente desde los territorios. Las regiones, por su parte, son los espacios definidos como territorios con afinidad geográfica, cultural, histórica, económica y social: identidad regional. Parten del concepto de Territorio/Proyecto (o proyectos de territorio) intentando rebasar las limitaciones socioeconómico-políticas que caracterizan al territorio físico como unidad administrativa.
\end{abstract}

30 Mientras que, en algunos casos -en especial en los pueblos con menor cantidad de habitantesexistieron procesos participativos en otros se operó con una lógica más centralizada a partir de la implementación de programas sociales verticalistas.

31 1. Corrientes-Riachuelo; 2 San Cosme-Itatí; 3 Goya-Esquina; 4 Saladas-Empedrado-Mburucuyá 5 Bella Vista-San Roque; 6

Chavarria - Mercedes; 7 San Miguel-Loreto; 8 ConcepciónSanta Rosa; 9 Sauce-Curuzú; 10 Monte Caseros-Mocorota; 11 Alvear-La Cruz; 12 Paso de los Libres-Tapebicuá; 13 ItuzaingóVilla Olivari; 14 San CarlosColonia Liebig; 15 Ita IbatéBerón de Astrada; 16 Santa LuciaLavalle; 17 Gobernador

Martínez-Yataity Calle-9 de Julio; 18 Santo Tome - Virasoro; 19 Pellegrini - Galarza. 


\section{CUADERNO}

Procesos de desarRollo local EN LA REgIÓN NEA: UnA APROXIMACIÓN TEÓRICA Y EMPÍRICA

32- El marco jurídico es la ley 25.300 , en cuyo enunciado propositivo se enfatiza la necesidad de estimular el fortalecimiento competitivo de las micro, pequeñas y medianas empresas que desarrollen actividades productivas en el país, mediante la creación de nuevos instrumentos y la actualización de los vigentes, con la finalidad de alcanzar un desarrollo más integrado, equilibrado, equitativo y eficiente de la estructura productiva.

33- Nodo 1: Capital, San Cosme, Itatí, Empedrado, San Luis, San Miguel, General Paz, Mburucuyá y Berón de Astrada.; Nodo 2: Concepción, Mercedes, Alvear y San Martín; Nodo 3: Monte Caseros, Curuzú Cuatiá y Sauce Nodo 4: Ituzaingó y Santo Tomé; Nodo 5: Goya, Lavalle, Esquina, Bella Vista, San Roque y Saladas.

34- Coincidimos en este diagnóstico con los resultados presentados por Alejandro Villar

(2004) en que se analizan diversas experiencias de Desarrollo Local en Argentina en los últimos diez años. promovidas desde el gobierno nacional, se presentan como una nueva institución que puede ser pública, privada o mixta y cuya función es orientar, previa planificación, el crecimiento y creación de nuevos proyectos que contribuyan al desarrollo productivo local, articulando y facilitando la utilización de instrumentos de apoyo a la competitividad de las empresas. La asignación de las agencias a cada uno de los municipios se encuentra condicionada a la capacidad de gestión o diligenciamiento de ellos, así como a los acuerdos políticos entre los gobiernos municipales, y el provincial y nacional. Pero, y aún cuando han sido designados los gerentes de forma consensuada por las Unidades de Coordinación, a la fecha no se han instrumentado acciones concretas. Del conjunto de localidades analizadas, se enmarcan en este tipo de procesos los municipios de Mercedes, Monte Caseros, Curuzú Cuatiá y Goya.

Finalmente, en la provincia se encuentra otra forma asociativa microrregional, pero de carácter interregional. Así bajo la denominación de Consorcio Centro Sur de Municipios, se integran algunos municipios de Corrientes (Curuzú Cuatiá, Monte Caseros y Mercedes) el de Chajarí (Entre Ríos), un municipio de Brasil (Uruguayana) y otro de Uruguay (Bella Unión). Con similares características, actualmente se está conformando otra microrregión integrada por los municipios de los departamentos de Lavalle, Goya y San Roque. Sin embargo, y si bien estos espacios parecen efectivamente posibilitar el encuentro e intercambio entre los dirigentes comunales y parecen haber contribuido a la gestión ordenada - y coordinada - entre municipios de las políticas sectoriales generadas a nivel nacional, los entrevistados señalan que han sido escasas las acciones programadas con carácter supra-local o regional.

Las principales debilidades que atentan contra la gestión concertada de tales espacios deberían buscarse, probablemente, en las insuficiencias jurídicas y normativas que regulan su funcionamiento, la escasa representatividad y poder de negociación que poseen frente a distintas instancias decisorias (gobierno provincial y nacional) y, al igual que en las microrregiones: la persistencia de una cultura localista (Villar,2004) (34 $^{34}$ municipalista que limita la concreción de proyectos de financiamiento compartido y la coexistencia tanto de perspectivas diferentes sobre el $\mathrm{DL}^{35}$, como de lógicas e intereses dispares en las intervenciones (la de los técnicos, la de los expertos, la de los funcionarios políticos, la de los representantes de la sociedad civil). 


\section{Ana María Pérez y Pablo Barbetti}

Procesos de desarRollo LOCAL EN LA REGIÓN NEA: UNA APROXIMACIÓN TEÓRICA Y EMPÍRICA

\section{A MODODE CONCLUSIÓN}

Independientemente de las perspectivas teóricas y las aproximaciones conceptuales desde donde se lo aborde, el enfoque del desarrollo local constituye, sin duda alguna, un paradigma que se ha instalado en nuestra región como un eje orientador del tratamiento de los problemas socio-económicos. El complejo dispositivo diseñado e implementado por los diversos organismos del Estado, que incluye la creación de nuevas instituciones, políticas e instrumentos específicos, da cuenta de la intención expresa de ellos en fortalecer procesos con tales características.

Un rasgo para destacar en ellos, en la región NEA, es la variedad de estrategias que se incluyen bajo la categoría de DL; a modo de enumeración general deberían acentuarse aquellas que se encuadran en los modelos del planeamiento estratégico, algunos ensayos con particularidades específicas de acuerdo a la localidad en las que se desarrollaron y finalmente, el conjunto de programas y proyectos sociales — diseñados en los niveles centrales de gobierno, aunque administrados a nivel municipal- y que se piensan destinados a impulsar el desarrollo local. Estas políticas han pretendido, en algunos casos, brindar atención a las cuestiones sociales, implementando acciones de promoción y desarrollo, con el fin de producir, en el corto plazo, modificaciones en la calidad de vida de los ciudadanos que sufren algún tipo de exclusión social y económica y generar movilización social con la acción productiva y la autogestión; mientras que en otros, han estado vinculados, más específicamente, con la esfera económica. Esta multiplicidad de objetivos y orientaciones pone de manifiesto cómo se han modificado y diversificado las funciones que actualmente se asigna a los municipios, sumándose al cuidado de la ciudad otras, vinculadas con diferentes perspectivas de desarrollo, así como la generación de emprendedores para el mercado.

Pero también dan cuenta de la permanencia de las principales ideas fuerza provenientes de la raíz neo-liberal, las que han continuado operando como orientadoras de estas políticas: estrategias compensatorias, criterios de focalización para la selección y atención de problemáticas y grupos específicos, descentralización y privatización de roles y funciones, configuran el modo operativo de estos proyectos, asentados en torno a la recupera-
35- Una entrevistada relataba que en el proceso de constitución de las microrregiones en la Provincia del Chaco se podía advertir claramente dos lógicas de intervención: una asociada al desarrollo local como desarrollo sustentable y sostenible y otra en la que se trabajaba a partir del marco teórico del desarrollo económico como eje de la propuesta. 


\section{CUADERNO}

Procesos de desarRollo local EN LA REgión NEA: UnA APROXIMACIÓN TEÓRICA Y EMPÍRICA

ción de lo local y que han sido asumidos, en parte, por los gobiernos provinciales y, en parte, por las distintas organizaciones de la sociedad. Algunas de estas ideas pueden encontrarse también en la etapa siguiente - durante la poscrisis - sirviendo, en unos casos, como plataforma para la gestión de gobierno (Programa de Regionalización y Desarrollo Local de la provincia del Chaco) o bien, como fundamento para el diseño de propuestas de gestión administrativa, u orientando las acciones de intervención territorial.

Al mismo tiempo, el análisis de estas experiencias, muestra que no son pocas las limitaciones que el modelo posee para que, en las prácticas concretas, se hagan efectivos varios de los supuestos que posibilitarían un mejoramiento real de las condiciones de vida de la población. En este sentido, algunas de las hipótesis iniciales de este trabajo, adquieren aún más relevancia: el carácter participativo de los espacios generados, la vinculación entre los resultados de los diagnósticos realizados y la complejidad de las problemáticas locales, el grado de pro-actividad de las políticas públicas con respecto a intereses y perspectivas de grupos y sectores tradicionalmente excluidos de la participación, constituyen los principales aspectos críticos de las intervenciones descriptas. Para Martínez Nogueira (op. cit.: 157), la debilidad de los diagnósticos, la ausencia de evaluaciones de factibilidad social y organizacional de las acciones propuestas y la insuficiente consideración de los procesos de gestión son algunos de los factores que, con frecuencia, permiten explicar las dificultades que se presentan en la implementación o la escasa apropiación social por parte de los actores locales.

Pero, y desde una mirada más comprensiva, debe destacarse que, además de las restricciones económicas, institucionales y sociales, propias de cada localidad, los fundamentos teóricos mismos sobre los que se asientan estas estrategias, derivan en modos de intervención en los que no se prioriza la gestión de cambios concretos, ni se garantizan desde los sectores que los promovieron- las condiciones políticas para que éstos se hagan efectivos.

En resumen, es poco lo que se ha avanzado en la concreción de una propuesta alternativa, en la que, como resultado de una construcción colectiva, se presenten experiencias innovadoras, se valore la democratización como condición concomitante del desarrollo o 


\section{Ana María Pérez y Pablo Barbetti}

PROCESOS DE DESARROLLO LOCAL EN LA REGIÓN NEA: UNA APROXIMACIÓN TEÓRICA Y EMPÍRICA

se planteen modelos organizativos que se aparten de las formulaciones clásicas del capitalismo, o presten particular atención a la recomposición de las relaciones entre lo económico y lo social. Por el contrario, y aun cuando, se podrían reconocer algunas experiencias más próximas al pensamiento de la economía solidaria, hasta el momento la gran mayoría de las respuestas elaboradas se han constituido más bien como paliativos frente a la crisis, centrando los esfuerzos más en la contención del malestar social y la preservación de la gobernabilidad que en encontrar soluciones a la pobreza o a propiciar espacios para la construcción de ciudadanía. En otros casos, las estrategias puestas en marcha se han orientado a consolidar aquellos sectores mejor posicionados y más próximos a los centros de poder y la economía.

De hecho, y como señalamos al inicio de este artículo, existen tanto en América Latina como en nuestro país, numerosos puntos sin resolver - muchos de ellos, directamente vinculados con las mínimas condiciones materiales de vida y trabajo- que operan como obstáculos e impiden el ejercicio democrático de la participación y la ciudadanía; pero, en cualquier caso, debería tenerse presente que la posibilidad de hablar de otro desarrollo en nuestras regiones, supone no sólo pensar en otras formas de organización económica, sino también "situar como eje de la propuesta la recuperación del papel de la producción de bienes públicos de alta calidad en la acción conjunta entre el Estado y la sociedad organizada" (Coraggio, op.cit: 13).

\section{BIBLIOGRAFIA}

CASTEL, R. (1997) La metamorfosis de la Cuestión social. Una crónica del salariado. Paidós. México, Barcelona, Buenos Aires.

CEPAL (1998): Gestión de Programas Sociales en América Latina. Volumen l. Serie de Políticas Sociales № 25. Naciones Unidas. Comisión Económica para América Latina y El Caribe. División de Desarrollo Social. Santiago de Chile.

CHOCONI, S (2003). Algunas ideas críticas sobre el Desarrollo Local: El caso de los consorcios productivos en la Provincia de Buenos Aires-Argentina. En Problemas del Desarrollo. Revista Latinoamericana de Economía. VOL. 34 № 135. X-XII 2003. 
Procesos de desarRollo local en la Región NeA: UnA APROXIMACIÓN TEÓRICA Y EMPíRICA

CLEMENTE, A. (2005) Políticas Sociales y alianzas para el desarrollo y la inclusión social. Lecciones del programa Fortal. En Clemente, A. Y Del Valle, (comp.): Pobreza, Desarrollo Y Alianzas Multiactorales. Balances y perspectivas. IIED-América Latina. Publicaciones.

CLEMENTE, A (2003) Descentralización y desarrollo en América Latina. Las contradicciones de una ecuación incompleta. En Desarrollo Local y Ciudadanía en América Latina. Retos para la cooperación Europea. Documento de trabajo de la Red de Cooperación Euro-Latinoamericana-RECAL - 07/2003. Madrid.

CORAGGIO, L (2004): Presentación para el público Latinoamericano. En LAVIILLE, J (comp.) Economía Social y solidaria. Una visión europea. Altamira. Buenos Aires.

DOMECQ, R. (2004) "Conocimiento, competitividad y calidad de vida" en Aprea, G (comp.): Problemas de comunicación y Desarrollo" Prometo. Buenos Aires.

EME, B, LAVILLE, JL (1994) Cohesión sociale et Emploi. Desclée de Brower. Paris.

GALLICCHIO, E (2004) El Desarrollo Local en América Latina. Estrategia política basada en la construcción de capital social. Programa de Desarrollo Local del Centro Latinoamericano de Economía Humana (CLAEH). Uruguay. Ponencia presentada en el Seminario "Desarrollo con inclusión y equidad: sus implicancias desde lo local". Córdoba. Argentina. Mayo2004.

GOLBERT, L (2004): “Notas sobre la situación de la historiografía sobre la política social en Argentina”. En BERTRANOU, PALACIO, SERRANO (comp.), En el país del no me acuerdo. Prometeo, libros, Buenos Aires.

KLISBERG, Bernardo (2002), Hacia una economía de rostro humano, Fondo de Cultura Económica, Argentina

LAVILLE, J.; SAINSAULIEU, R. (Dir). (1997) Socilogie de association. Des organisations a lépreuve du changement social. Desclée de Brower. Paris.

LAVIILLE, J (comp.). (2004): Economía Social y solidaria. Una visión europea. Altamira. Buenos Aires.

LLORENS, J. L; ALBURQUERQUE, F y DEL CASTILLO, J (2002). Estudio de casos de desarrollo económico local en América Latina. Banco Interamericano de Desarrollo. Serie de informes de buenas prácticas del Departamento de Desarrollo. Washington, D. C. http:/ /www.iadb.org/sds/doc/desarrolloeconomicolocal.pdf.

MARTINEZ NOGUEIRA, R. (2004) Historia, aprendizaje y gestión pública: las políti- 


\section{Ana María Pérez y Pablo Barbetti}

Procesos de deSARROLLO LOCAL EN LA REGIÓN NEA: UNA APROXIMACIÓN TEÓRICA Y EMPÍRICA

cas dirigidas a la pobreza en el Estado remanente. En Bertranou y otros (comp.): En el país del no me acuerdo. (Des) memoria institucional e historia de la política social en la Argentina. Prometeo. Buenos Aires.

MAUSS, M. (1923): Essai sur le don. Forme et raison de l'echange dans les sociétés archaïques. Année sociologique, Paris.

PANAIA, M (2004) (Coord.): "Crisis Fiscal, Mercado de Trabajo y Nuevas Territorialidades en el Nordeste Argentino" Editorial La Comena. Buenos Aires

PERROUX, F (1960): Economie et societé, contrainte-échange-don .Presses Universitaires de France. Paris.

POLANYI, K. (1983): La gran transformación. Fondo de Cultura Económica.

PUTMAN, Robert (1994), Para hacer que la democracia funcione, Editorial Galac, Caracas.

RAZETO MIGLIARO, L. (1988): Economía de solidaridad y mercado democrático, Libro tercero. Fundamentos de una teoría económica comprensiva. Programa de Economía del Trabajo. Santiago de Chile.

ROFMAN, A. Y otros (2004): La dimensión de la cultura y la comunicación en los procesos de desarrollo local. en Aprea, G (comp.): Problemas de comunicación y Desarrollo" Prometo. Buenos Aires.

ROSANVALLON, P. (1998): La nueva cuestión social. Repensar el estado de providencia. Ed. Manantial. Buenos Aires

SALAMON, L, ANHEIER, H (1995): Defining the Nonprofit Sector. Manchester University Press. Manchester.

VILLAR, A. (2004): Una década de desarrollo local: Balances y Perspectivas. En Revista Virtual Mundo Urbano № 24. Universidad Nacional de Quilmes. Buenos Aires. 
